\title{
Old vaccines, new diseases: when BCG meets SPPL2a
}

In the current issue of Nature Immunology, Casanova and colleagues demonstrate that humans (and mouse models) with autosomal-recessive SPPL2a deficiency have a severe defect in conventional dendritic cell 2 survival and production of IL-12 and IL-23, and diminished IFN- $\gamma$ secretion by mycobacterium-specific memory $T$ cells, thus resulting in increased susceptibility to mycobacterial diseases.

\section{Sergio D. Rosenzweig}

\begin{abstract}
$\mathrm{n}$ the current issue of Nature Immunology, a study from the Casanova laboratory provides yet another surprise by adding a new metabolic pathway associated with Mendelian susceptibility to mycobacterial diseases $(\mathrm{MSMD})^{1}$. This term, also coined by the Casanova lab, describes defects in production of the cytokine IFN- $\gamma$ or in IFN- $\gamma$ responsiveness that lead to a particular vulnerability to mycobacterial infections. In this manuscript, the authors report two families with MSMD associated with autosomal recessive (AR) mutations in SPPL2A. This gene encodes the protease responsible for the cleavage of the N-terminal fragment (NTF) of CD74, the human leukocyte antigen invariant chain in major histocompatibility complex (MHC) class II molecules (Fig. 1). Overaccumulation of CD74 NTF in both myeloid and lymphoid antigen-presenting cells appears to be exquisitely toxic to $\mathrm{CD} 1 \mathrm{c}^{+}$conventional dendritic cells ( $\left.\mathrm{cDC} 2 \mathrm{~s}\right)$,
\end{abstract} thus leading to the targeted depletion of cells producing the interleukins IL-12 and IL-23. In addition, mycobacterium-specific IFN- $\gamma$ production by SPPL2a-deficient memory $\mathrm{T}_{\mathrm{H}} 1^{*}$ cells is impaired. This situation creates a 'perfect storm' leading to MSMD by creating a milieu of low IFN- $\gamma$ -inducer cytokines such as IL-12 and IL-23, which would otherwise be produced by specific dendritic cells, together with low IFN- $\gamma$ production by $\mathrm{T}$ cells in response to mycobacterial challenge.

To further reinforce the relevance of the role of the SPPL2-CD74-NTF pathway in mycobacterial control, the authors took the extra (and excellent) step of reevaluating a mouse model with an Sppl2a deficiency. Of note, several Sppl2a-defective mouse models have been described. All show a striking and consistent impairment in dendritic cell and B cell development, the latter of which results in abnormal immunoglobulin production and antibody function ${ }^{2-5}$.

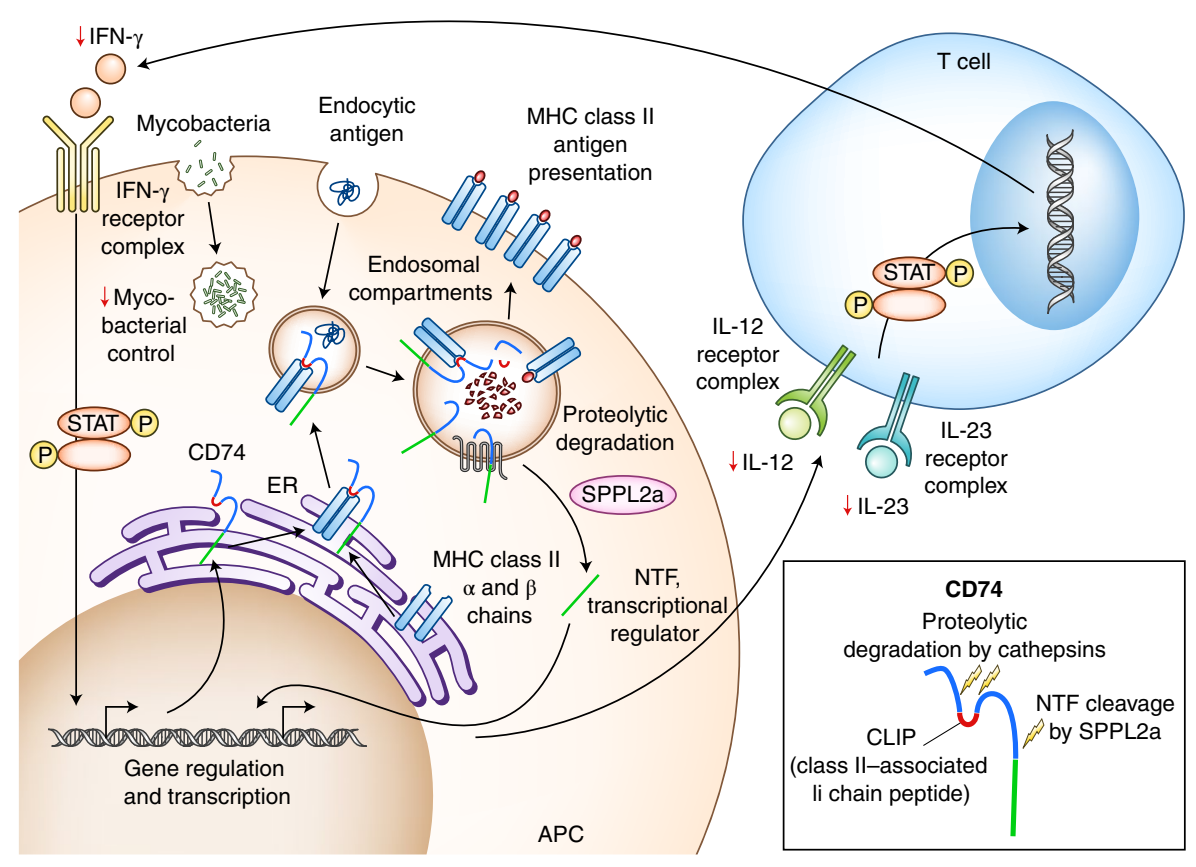

Fig. 1 | CD74, MHC class II, SPPL2a, antigen-presenting cells and T cells. CD74 (also known as invariant chain or li) is a transmembrane glycoprotein that binds the MHC class II $\alpha$ and $\beta$ chains in the endoplasmic reticulum (ER); facilitates delivery of this multimeric complex to specialized endosomal compartments; and is associated with the antigen-presentation process. The class II-associated li chain peptide (CLIP) segment in CD74 is the major interaction site with the MHC class II and prevents its premature binding to antigenic peptides. While endocytic antigens are being processed, the degradation of CD74 is also initiated by cathepsins (inset). IFN- $\gamma$ signaling through phosphorylated (P) STAT1 dimers stimulates CD74 expression as well as the cathepsins involved in its metabolism. SPPL2a, the intramembrane protease cleaving the CD74 NTF, completes the CD74 cycle by releasing this domain into the cytoplasm. In turn, this peptide can be translocated into the nucleus, where it acts as a transcriptional regulator. Casanova and colleagues ${ }^{1}$ demonstrate that humans (and mouse models) with AR SPPL2a deficiency have a severe defect in CDC2 survival and IL-12/IL-23 production; diminished IFN- $\gamma$ secretion by mycobacterium-specific memory $T$ cells; and elevated susceptibility to mycobacterial diseases (red arrows). APC, antigen-presenting cells (representing macrophages and/or dendritic cells).

As previously reported, Casanova and colleagues $^{1}$ found that mice defective in Sppl2a expression or function were devoid of cDC2s. In addition, the authors found that T cell production of IFN- $\gamma$ was diminished, and the animals were also susceptible to mycobacterial infections by both highervirulence (Mycobacterium tuberculosis) and lower-virulence (Mycobacterium bovis (BCG)) bacteria, much like the AR 
SPPL2a-deficient patients studied in their laboratory. Interestingly, and in contrast to the findings in SPPL2a-deficient mice, no clinically meaningful B cell defects were detected among the patients. Altogether, these features highlight the critical role of SPPL2a in mycobacterial control as well as the usefulness of studying animal models in the characterization of new primary immunodeficiencies (PIDs). However, in this process, it is important to recognize the potential for the existence of ortholog differences across species as well as allelic variants and clinical heterogeneity ${ }^{6}$. In the case of human SPPL2a deficiency, the features of the human disease reported in these three individuals resembled those of the mouse models (or vice versa) but differed with regard to the effects on the B cell compartment. What accounts for this difference between species remains unclear.

If we think of the manuscript by Casanova and colleagues ${ }^{1}$ as a suspense novel (well written, complex and captivating, if I may), as critical readers of the plot, at the end we might still be expecting more (a sequel?) to solve the remaining mysteries and reveal more about AR SPPL2A deficiency, the new killer on the block of MSMD. Which mechanism is involved in AR SPPL2A deficiency that makes it such a lethal killer to $\mathrm{CDC} 2 \mathrm{~s}$, and what makes cDC2s so exquisitely susceptible to CD74NTF accumulation that they disappear from the story even before their very first act? ( $\mathrm{cDC} 2$ progenitors were also absent from peripheral blood in the patients.) The authors leave us some hints to consider while awaiting those answers: is the unfolded-protein response or endoplasmic reticulum-associated degradation involved in the cDC2 crime scene? Because CD74 assists in the folding and trafficking of MHC class II molecules through the endoplasmic reticulum, it is perfectly reasonable to suspect that these mechanisms may be necessary participants in the plot? Moreover, does the diminished expression of IFN- $\gamma$ play a dual role in this scenario, on the one hand not delivering enough of the death-sentence message to macrophages, the natural final executers of mycobacteria, and on the other hand decreasing expression of CD74, a chaperone and trafficking adaptor for MHC class II expression, another IFN- $\boldsymbol{\gamma}$-responsive protein necessary to mycobacterial control ${ }^{1,5}$ ? In any case, there are still plenty of remaining mysteries in the SPPL2a-mycobacterial susceptibility conundrum that colleagues in the field will need to solve, hopefully sooner than later, because the intrigue is building!

Another interesting aspect of this paper, although not directly derived from its elegant experimental design and results, relates to the patients' clinical phenotype. The three patients described in this report presented with BCG-associated disease. These complications (again) alerted our colleagues about PIDs in general and MSMD in particular, and in this case led to the description of this novel AR SPPL2a deficiency. Moreover, most MSMD-related genes and diseases were originally described in patients presenting with BCG disease ${ }^{8,9}$. But, to be clear, this is not an 'experiment of nature' as Robert Good described PIDs almost 50 years $\mathrm{ago}^{10}$ : BCG complications after newborn vaccination are iatrogenic infections, which are life threatening for certain PIDs and are probably preventable. In addition, attention to early BCG vaccination has experienced an unprecedented, once-in-a-lifetime interest in recent years. This resurfaced focus on BCG does not exactly correlate with increased efficacy in preventing tuberculosis, the original aim driving Alfred Calmette and Camille Guérin's 1908-1921 attenuated vaccine development (reviewed in ref. ${ }^{11}$ ). There is a growing body of evidence associating early BCG vaccination with a heterologous immune-stimulatory effect leading to protection against lifethreatening infectious diseases unrelated to tuberculosis and even atopy ${ }^{12,13}$. As a medical community interested in genetic susceptibility to mycobacterial infections, we have gained remarkable insights from BCG complications in PIDs, instructing us about the mechanisms for mycobacterial infection control-a kind of "when life gives you lemons, make lemonade' situation. Fortunately, multiple approaches are under consideration in an attempt to prevent BCG complications. Some of them are as simple as delaying BCG vaccination for 2-6 months after delivery to allow patients to show signs of immunodeficiency before the vaccine is given, and others are currently used in countries conducting newborn screening for severe combined immunodeficiency (another group of PIDs highly susceptible to infections by mycobacteria, among other pathogens) $)^{14,15}$. In addition, there is active ongoing research on developing minimally harmful and highly efficient antituberculosis vaccines ${ }^{16}$, as Calmette and Guérin envisioned more than a century ago. Let us hope that the next gene associated with MSMD is described in patients who did not have to receive $3.75 \times 10^{5}$ to $3.2 \times 10^{6}$ live attenuated BCG intradermally within their first month of life to alert us about their vulnerability and frail immune systems ${ }^{11}$.

In summary, the paper by Casanova and colleagues ${ }^{1}$ is another great example of this lab's effort to decipher the mysteries behind unsolved cases of MSMD. To date, these efforts have expanded knowledge on immunity to mycobacteria beyond the usual suspects (IFNGR1, IFNGR2, STAT1, $I L 12 R B 1$ and $I L 12 B$, among others), now involving less obvious pathways and more cell-type-specific defects increasing susceptibility to mycobacterial diseases.

\section{Sergio D. Rosenzweig \\ Immunology Service, Department of Laboratory \\ Medicine, NIH Clinical Center, Bethesda, MD, USA. \\ e-mail:srosenzweig@cc.nih.gov}

Published online: 20 August 2018

https://doi.org/10.1038/s41590-018-0193-0

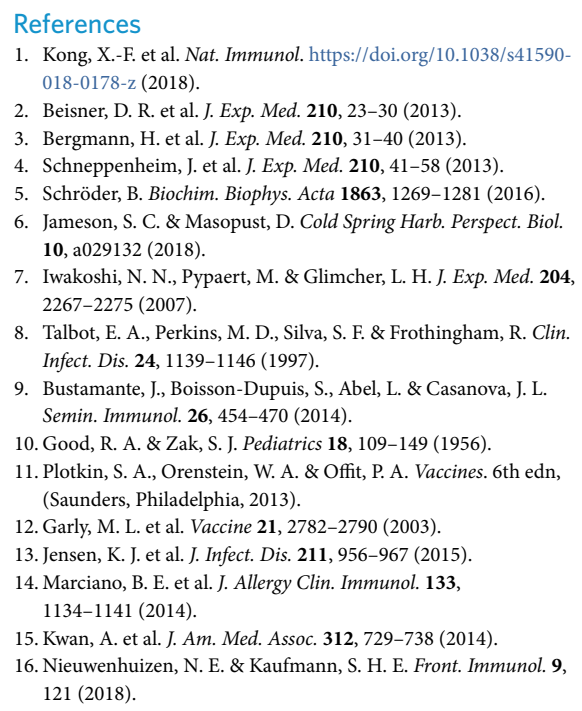

Acknowledgements

This work was supported by the Intramural Research Program, NIH Clinical Center, US National Institutes of Health (NIH). The content of this article does not necessarily reflect the views or policies of the Department of Health and Human Services, nor does mention of trade names, commercial products, or organizations imply endorsement by the US government.

Competing interests

The author declares no competing interests. 